\title{
When working memory is in a mood: Combined effects of induced affect and processing of emotional words
}

\author{
Katarzyna Rączy ${ }^{1}$ D . Jarosław Orzechowski ${ }^{1}$
}

Published online: 12 March 2019

(C) The Author(s) 2019

\begin{abstract}
It is already well established that the working memory system can be influenced by moods or emotional stimuli. However, the exact combined impact of these two on the performance of working memory remains a puzzle. To examine the effect that the emotional content of stimuli has on working memory performance, 90 participants performed a 2-back task with emotional content (positive, neutral, and negative words) when they were in a positive, neutral, or negative mood. Repeated-measures ANOVA with mood as between-subjects factor and emotional load as within-subjects factor revealed a main effect of emotional content for both performance accuracy and reaction times in a 2-back task, and a main effect of mood for performance accuracy. Participants reacted significantly faster to negative words independently of their mood state. They were significantly more accurate when they were in the positive mood, but when they processed positive words they were less accurate. Additionally, to test whether loading the working memory system can reset the combined effect of mood and emotional stimuli, we measured the participants' mood before and after they performed the n-back task; this revealed a significant effect of the n-back task on the mood. Together, these results suggest that although mood and emotional content do have a robust effect on working memory, in some instances combining them does not heighten their individual effects. Moreover, the results also show that participants might be easily distracted from moods by simply performing an n-back task.
\end{abstract}

Keywords Mood $\cdot$ Working memory $\cdot$ Limited capacity $\cdot$ Emotional content

\section{Introduction}

A 1960s TV series called "Star Trek" had a very distinctive character: Spock. Besides his very interesting looks, what mainly set him apart from the humans was the fact that neither moods nor emotions had an impact on his cognition, actions, or decision-making (Forgas 2000). Because of this huge role that emotions play in our lives, psychology has devoted a great deal of attention to how mood affects the working memory system. Working memory is understood as the "ability to mentally maintain information in an active and readily accessible state, while concurrently and selectively processing new information" (Conway et al. 2007, p. 3). "This concept and its limits is a key part of the human condition. [...] We need working memory too in language comprehension, [...]; in

Katarzyna Raczy

raczy.katarzyna@gmail.com

1 Psychology Department, Jagiellonian University, Ingardena 6, 30-060 Kraków, Poland arithmetic, [...]; in reasoning, [...]; and in most other types of cognitive tasks". (Cowan 2005, p. 2). If mood may moderate the influence of emotion on working memory functioning, it seems extremely important to properly understand the complexity of its relations. The disparate impact of mood on cognitive functions has been repeatedly shown in several experiments (e.g. Donovan and Kleinknecht 2007; Teasdale 1983; Carpenter et al. 2007). For example, a positive mood improves the performance of some executive functions, such as planning, switching between tasks, and refreshing (Phillips et al. 2002); on the other hand, negative mood has been shown to impede performance in tests of problem-solving, working memory, and attention (Cheng and Holyoak 1985; Spies et al. 1996). Although the multiplicity of research complies with the assumption that mood plays a key role in the functioning of the working memory system, the exact effect on its functioning is still not clear. There are several significant approaches to the subject.

Mood, regardless of its type (negative or positive), worsens the functioning of working memory (Eysenck and Calvo 1992). When mood-congruent information is retrieved, regardless of the mood type, or when subjects process intrusive 
thoughts or worries (Eysenck and Calvo 1992; Seibert and Ellis 1991) that involve working memory capacity, task performance deteriorates in contrary to the working memory performance when participants are in the neutral mood.

On the other hand, mood plays an informative role in the working memory system. A negative mood is seen as a mechanism that evolved to help anticipate potential dangers; therefore, it is treated as a warning sign that triggers careful examination and analysis of the immediate surroundings (Batson et al. 1992). While a negative mood seems to trigger analytical thinking, positive mood, which reflects a less stressful environment, worsens task performance. Positive mood also impairs certain working memory functions, such as planning, reasoning, or assessing (Oaksford et al. 1996; Seibert and Ellis 1991; Spies et al. 1996) because it triggers heuristic thinking. There are reports, however, that a positive mood has a facilitative impact on working memory processes (Isen 1999; Ashby et al. 1999). Performance on certain tasks significantly improves when participants are in a positive mood: it facilitates creativity and flexibility and improves moodcongruent recall of memories (Isen et al. 1987; Teasdale and Fogarty 1979).

It is currently an open question as to whether the modulation of working memory by mood occurs when the emotional content of external stimuli is also manipulated. This could affect what information is attended to (e.g., particular stimuli or their attributes). The emotional content of stimuli is known to affect the distribution of attention, such that emotional stimuli are likely to capture and hold attention (Pratto and John 1991; Reimann and McNally 1995) and gain prioritized processing (Anderson and Phelps 2001; Dolan 2000; Tabert et al. 2001). Depending on task requirements, attentional biasing toward emotional content could also have a detrimental effect on working memory performance, especially when a participant is in a certain mood. Processing emotional content may result not only in attending to emotional stimuli over nonemotional stimuli, but also in a specific way of focusing attention on the stimulus because mood impacts working memory performance. Although emotion-relevant stimuli usually capture and hold attention, how working memory capacity is distributed when a participant is in a certain mood remains an open question.

Recently researchers have focused on the processing of written emotional words. Emotional words evoke differential responses than neutral ones, however regardless of the emotional content they are often processed within a context: of the prior paragraph or the inner emotional state such as mood. Indeed the reader's mood affects the recognition of emotion words. In line with the mood congruency argument proposed by Bower (1981), the processing is easier when the mood is congruent with the emotional tone of the information, thus suggesting a strong connection between people's mood and their cognitive processes. When discussing the effects that a certain mood has on the recognition of emotionally-loaded words, research has repeatedly shown that words that are not congruent with the mood tend to be facilitated relative to the ones that are incongruent with the mood (e.g., Small 1985; Halberstadt et al. 1995; Niedenthal and Setterlund 1997; Olafson and Ferraro 2001; Ferraro et al. 2003; Sereno et al. 2015), However, in their experiment Sereno and colleagues (Sereno et al. 2015) showed a significant interaction between participant mood and word emotionality when they performed lexical decision task, the reported effects were not consistent with mood-congruency effects. While positive and negative mood facilitated responses regardless of emotional content of a word, neither positive nor negative mood additionally facilitated responses to mood-congruent words.

Thus, here we aimed to shed an additional light on the ongoing debate whether the mood has a facilitatory impact on processing mood-congruent words within a working memory system. Here, we used a 2-back task with positive, negative and neutral words to establish whether we would observe a combined effect of the mood and mood-congruent words. Alternatively the modulation of working memory would occur due to the mood manipulation itself regardless of the emotional content of the words.

Regardless of its type, mood burdens working memory capacity. In order to gain attention, a new incoming stimulus competes for resources with the stimuli already being processed. As more capacity is needed to perform a complex working memory task, less capacity is left to sustain the negative mood. Therefore, its limited capacity facilitates distraction from the negative mood. Van Dillen and Koole (2007) showed that performing a working memory task may distract an individual from a negative mood, can reduce anger (Gerin et al. 2006; Rusting and Nolen-Hoeksema 1998), or even decrease symptoms of depression in depressed participants (Joormann and Siemer 2004; Morrow and Nolen-Hoeksema 1990). This replacement of the mood seems to work only when participants are in a negative mood but not in the positive one (Van Dillen and Koole 2007). It could be due to the different nature of the positive and negative mood. However, if the mood were treated as incoming information that has to be processed by the working memory system, mood, regardless of its type would be displaced from the working memory system. To further test the notion of limited capacity of the working memory system, we tested whether the participants could be distracted from a negative and positive mood after performing an n-back task. If both - negative and positive mood, overload the working memory system, they would be reduced to the neutral mood after performing an n-back task.

There are several ways to manipulate the mood of the participants. For example, by having them listen to either "happy" or "sad" music, or by describing either "happy" or "sad" memory. Although techniques used so far to manipulate the mood state have resulted in expected, either positive or 
negative, mood state of the subject, what events participants actually recall or whether the music is as sad for everyone, seems rather difficult to measure.

Thus, in our study, we decided to evaluate another potential tool aiming at mood manipulation, i.e. the Cyberball paradigm. Cyberball, the virtual analogue to the ball-tossing paradigm introduced by Williams et al. (2000), was commonly used to study ostracism. During the online game participants believe they are playing with two or three other participants, while they are actually playing with the computer fully controlled by the experimenter (Williams and Jarvis 2006). In the ostracize condition, the participants receive the ball only few times at the beginning of the procedure, while in the inclusion condition they receive the ball regularly throughout the whole game. Several studies using the Cyberaball demonstrated significantly higher level of distress after being excluded from the game (Eisenberger et al. 2003), and a decrease in reported mood when being ostracized (Williams 2007; Ruggieri et al. 2013). Also, an fMRI study showed increased brain activity similar to physical pain (Eisenberger et al. 2003). In Cyberball, manipulation of the subjects' mood is made possible by manipulating only one parameter - i.e. the number of balls tossed. Thus, here we decided to use the Cyberball instead of other mood manipulations.

\section{Pilot Study}

\section{Method}

We first conducted a pilot study in order to establish which of the three-intensity mood manipulations would have the greatest impact on mood fluctuations. We also tested the participants' performance accuracy in the 3-back task.

Participants Ninety participants (age: $\mathrm{M}=21.12 ; \mathrm{SD}=2.01$; 29 males) who were mostly undergraduates (not psychology students) at Jagiellonian University took part in the experiment. Participants were randomly assigned to one of the three mood conditions (positive $(n=30)$, neutral $(n=30)$ or negative $(n=30)$ ) and to one of the three intensity conditions (low $(n=30)$, intermediate $(n=30)$ or high $(n=30))$. They did not receive any course credit or monetary compensation for their participation.

\section{Materials and Procedure}

Mood To manipulate mood, we used the Cyberball paradigm (Williams et al. 2000) with three different intensities. The original settings (inclusion vs ostracism) were used to manipulate neutral and negative moods, respectively. However, we also added a third condition (positive), in which the ball was tossed only to the participant during the whole procedure.
Thus, in the negative condition participants received the ball only twice, in the neutral condition 7 times and in the positive condition 15 times. The total number of balls being tossed (between the participant and the other players), regardless of the condition, was 30 . The intensity of the manipulation differed among the participants. In the low-intensity condition, participants were told that they were playing with two coplayers via the Internet. In the medium- and high-intensity conditions, they played with the two participants sitting next to them, who were actually the experimenter's male and female assistants, while the participants played with the computer. During the whole experiment participants were asked to complete several questionnaires. Below we provide a description of the questionnaires used in the study.

Questionnaires Participants completed questionnaires relating to their mood state at three-time points: before and after the Cyberball procedure, and at the end of the study (after performing the n-back task). In all intensity conditions participants were asked to answer the same question: "Which mood state are you in now?" Participants answered on a 7-point scale (ranging from 0 - very negative to 6 - very positive) (See Fig. 1 b). Additionally in the high intensity condition, before playing Cyberball participants were asked to answer 15 questions concerning their mood state (e.g. "Are you sad?") and answered on a 7-point scale (ranging from 0 - very negative to 6 - very positive). After playing the game they were asked to additionally answer three open questions: 1) How did you like the game? 2) What was the game about? 3) How did you feel during the game?

This allowed an evaluation of participants' mood fluctuations throughout the experiment.

N-Back The n-back task was performed on a 15 -in. computer screen with a resolution of $1366 \times 768$ and programmed in PsychoPy v1.80 (Peirce, 2009); a grey background and a black font were used for the instructions as well as the stimulus. The words used in the experiment differed in the type of emotional load (positive, negative, neutral). Prior to the pilot study, all words were evaluated by fifteen independent participants (mostly undergraduates at the Jagiellonian University, mean age 25 years, range 20-29) on a 5-point scale (ranging from $-2-$ very negative to $+2-$ very positive). This allowed the choice of 72 words, allocated into three groups of 24 . The n-back task had 2-back, 3-back and 4-back sets. Each set was also divided into three emotional loads: negative, positive, and neutral. In every n-back variant (2-back, 3-back, and 4-back) there was an equal number of emotional words of different types ( 8 negative, 8 positive and 8 neutral). Every word was displayed for $1000 \mathrm{~ms}$ with a $500 \mathrm{~ms}$ interval. Here we used a 3-back as a rule. During all this time $(1500 \mathrm{~ms})$, participants responded whenever the current stimulus was the same as the one presented 3 positions back in the sequence. The stimuli 
were presented sequentially. Every participant performed the exact same n-back task (with a pseudorandom order) (see Fig. $1 \mathrm{a}$ ). We decided to present stimuli sequentially for two reasons. A crucial thing in emotional and mood processing is how the attention is distributed and shifted, thus sequential way of presenting stimuli was the best option to test which stimuli would gain attention and prioritized processing, the effect that would not have been achieved if using a block design. Secondly, mood can be vague and disappear quickly, so our aim was to ensure that every stimuli category has the same impact on the mood and vice versa.

\section{Results}

Statistical analyses were conducted with IBM SPSS Statistics 21.

Mood Manipulation To test which experimental manipulation worked best, we ran paired t-tests between mood assessment before and after playing Cyberball. In the negative mood condition, with both intermediate and high intensity, the difference between the two measurements was significant $(\mathrm{t}(9)=$ $9.000, p<0.001 ; \mathrm{t}(9)=8.232, p<0.001$, respectively). There was no difference in the low intensity condition $(\mathrm{t}(9)=0.688$, $p=0.509)$. In the neutral condition only the one with high intensity was significant $(\mathrm{t}(9)=3.674, p=0.005$; low and intermediate intensity were not significant $(\mathrm{t}(9)=1.616$, $p>=0.140, \mathrm{t}(9)=1.000, p=0.343$, respectively). In each of the neutral intensity conditions, the average answer on the Likert scale varied from 3 to 4 (neutral to a rather positive mood). In the positive condition we found a significant difference only in the high intensity variant $(\mathrm{t}(9)=-2.400, p=$ $0.040)$. We failed to obtain significant results in the remaining low and intermediate intensities $(\mathrm{t}(9)=-1.238, p=0.247$, $\mathrm{t}(9)=-0.461, p=0.656$, respectively; see Table 1).

a

\section{2-back task}

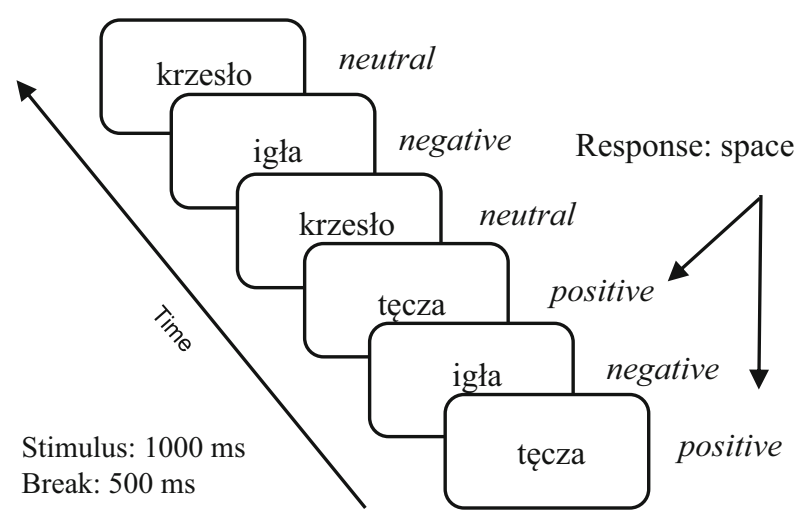

Fig. 1 a 2-back task with three experimental conditions: positive, negative and neutral. Stimuli used in the experiment: e.g. neutral: krzesło (chair), okno (window), stół (table), negative: igła (needle), zabójstwo (murder), narkotyki (drugs), positive: tęcza (rainbow), niebo
Therefore, considering that only high intensity manipulation turned out to be significant in all mood conditions, we decided to use the high intensity manipulation in the main study.

N - Back Task Participants performed a 3-back task after each mood manipulation (positive, negative, neutral) in all intensities (low, intermediate, high) with emotional (positive, negative) and neutral words. The accuracy of the given answers in this task was $43 \%(\mathrm{SD}=0.19)$.

\section{Discussion}

We tested whether the Cyberball paradigm (Williams et al. 2000), which differs across each condition in only one parameter, that is the number of balls tossed, could be successfully used to manipulate participants' moods. The results showed a significant effect of mood manipulation in the high-intensity condition. Thus, in the following Main Experiment, we applied only the high-intensity condition.

Performance accuracy in the 3-back task was below the chance level; in the following experiment we used the same n-back task with the only difference that participants were asked to react with a 2 -back rule.

\section{Main Experiment}

In the main experiment we tested whether the modulation of working memory by mood occurs when the emotional content of external stimuli is also manipulated, i.e. whether there would be an interaction between mood and emotional content of words, or alternatively no combined effect of those two factors. Our reasoning here was that if there is a combined effect of mood and emotional content of stimuli, the effects

b

$$
\text { Experimental Design }
$$
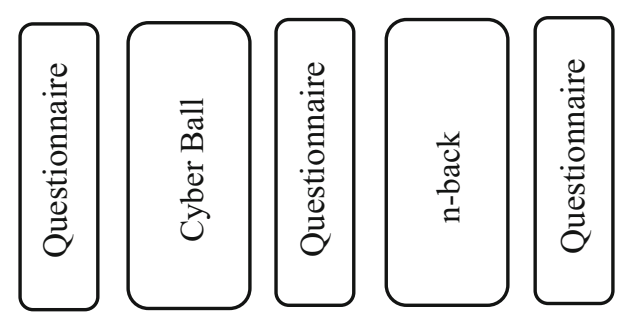

Time

(heaven), marzenie (dream). b Experimental Design: participants completed questionnaires before and after playing Cyberball and at the end of the study i.e. after performing 2-back task 
Table 1 Mood Manipulation before and after playing Cyberball in all three intensity conditions. Participants answered the question: "Which mood state are you in now?" on a 7-point scale $(0$ - very negative to $6-$ very positive). Significance levels: $* p<0.05, * * p<0.005$, $* * * p<0.001$

\begin{tabular}{|c|c|c|c|c|c|c|}
\hline \multirow[t]{2}{*}{ Manipulation of the Mood State } & \multirow[t]{2}{*}{$\mathrm{N}$} & \multicolumn{2}{|l|}{ M } & \multicolumn{2}{|l|}{$\mathrm{SD}$} & \multirow[t]{2}{*}{$\mathrm{t}$} \\
\hline & & before & after & before & after & \\
\hline \multicolumn{7}{|l|}{ Low-intensity Condition } \\
\hline Negative mood before vs after Cyberball & 10 & 4.70 & 4.50 & 0.82 & 0.71 & 0.688 \\
\hline Neutral mood before vs after Cyberball & 10 & 4.30 & 3.70 & 0.95 & 0.48 & 1.616 \\
\hline Positive mood before vs after Cyberball & 10 & 3.70 & 4.50 & 1.77 & 0.97 & -1.238 \\
\hline \multicolumn{7}{|l|}{ Medium-intensity Condition } \\
\hline Negative mood before vs after Cyberball & 10 & 4.90 & 2.80 & 0.99 & 0.79 & $9.000^{* * * *}$ \\
\hline Neutral mood before vs after Cyberball & 10 & 4.60 & 4.00 & 1.51 & 1.56 & 1.000 \\
\hline Positive mood before vs after Cyberball & 10 & 3.90 & 4.20 & 1.20 & 1.40 & -0.461 \\
\hline \multicolumn{7}{|l|}{ High-intensity Condition } \\
\hline Negative mood before vs after Cyberball & 10 & 4.80 & 1.60 & 1.31 & 1.06 & $8.232^{* * * *}$ \\
\hline Neutral mood before vs after Cyberball & 10 & 4.10 & 3.50 & 0.88 & 0.97 & $3.674^{* *}$ \\
\hline Positive mood before vs after Cyberball & 10 & 4.40 & 5.50 & 1.26 & 0.53 & $-2.400^{*}$ \\
\hline
\end{tabular}

of mood and processing emotional words would be strengthened. For example positive words in the positive mood would be processed slower than positive words in the neutral mood. On the other hand, negative words combined with the negative mood would be processed faster than those in the neutral one. Alternatively, mood and emotional content would be processed independently, and we would not observe a significant interaction.

In the main part of the study, we further tested whether mood, regardless of its type, loads working memory, and whether these effects could be reset by simply performing a working memory task. We tested whether loading working memory (by using the n-back task) can attenuate the effects of positive as well as negative moods.

\section{Method}

Participants Ninety participants (age: $\mathrm{M}=21.42$; $\mathrm{SD}=1.92$; 52 males) who were mostly undergraduates (but not psychology students) took part in the experiment. Participants were randomly assigned to one of the three conditions: positive $(n=30)$, neutral $(n=30)$ or negative $(n=30)$ mood groups. Participants received chocolate bars for taking part in the study.

\section{Materials and Procedure}

Mood The high-intensity mood manipulation with the Cyberball paradigm was used.

Questionnaires Participants completed all questionnaires assigned to the high-intensity mood group. Before playing Cyberball they were asked to complete a 15-item questionnaire concerning their mood state, and afterwards to answer three open questions concerning the game. After each stage they additionally answered the question "Which mood state are you in now?" (see Questionnaires section above).

$\mathrm{N}$-Back The n-back procedure was the same as in the pilot study, with the only difference that participants were instructed to react to a 2-back rule.

\section{Results}

Statistical analyses were conducted with IBM SPSS Statistics 21.

Mood Manipulation Paired t-test analyses were conducted for the evaluation of the mood before and after playing Cyberball. It showed that the experimental manipulation was successful in the positive $(\mathrm{t}(29)=-2.262, p=0.031)$, negative $(\mathrm{t}(29)=$ $11.276, p<0.001)$, and neutral condition $(\mathrm{t}(29)=6.440$, $p<0.001$; see Table 2). After playing Cyberball, in a positive condition, the mood of the participants significantly increased, in a negative condition it significantly decreased, and in a neutral one it significantly decreased, however remained on the neutral level and was averaged as 3.57 (see Table 2).

N - Back Task We first tested the accuracy of the performance in the given 2-back task. The accuracy was calculated separately for each participants, and we decided to exclude three participants from the final analysis due to their performance accuracy in the 2-back task being below the chance level and more than 3 standard deviations below the mean accuracy of the group $(38 \%, 34 \%$ and $17 \%)$. Thus, the final analysis included 87 participants. The accuracy of the performance was $72 \%$ ( $\mathrm{SD}=3.33$ ). First, we ran two-way repeated measures ANOVA for RTs (reaction times) in the 2-back task for 
Table 2 Mood Manipulation in all experimental conditions. Participants answered the question: "Which mood state are you in now?" on a 7-point scale $(0-$ very negative to 6 - very positive). Significance levels: $* p<0.05, * * p<0.005, * * * p<0.001$

\begin{tabular}{|c|c|c|c|c|c|c|}
\hline \multirow[t]{2}{*}{ Manipulation of the Mood State } & \multirow[t]{2}{*}{$\mathrm{N}$} & \multicolumn{2}{|l|}{ M } & \multicolumn{2}{|l|}{ SD } & \multirow[t]{2}{*}{$\mathrm{t}$} \\
\hline & & before & after & before & after & \\
\hline Negative mood before vs after Cyberball & 30 & 4.67 & 2.77 & 0.76 & 0.63 & $11.276^{* * * *}$ \\
\hline Positive mood before vs after Cyberball & 30 & 4.37 & 4.77 & 0.67 & 1.01 & $-2.262^{*}$ \\
\hline Neutral mood before vs after Cyberball & 30 & 4.63 & 3.57 & 0.85 & 0.68 & $6.440^{* * *}$ \\
\hline
\end{tabular}

emotional load conditions (positive, neutral, negative) as within-subjects factor and mood conditions (positive, neutral, negative) as between-subjects factor. This ANOVA revealed a significant effect of the emotional load [548, 529, $471 \mathrm{~ms}$ for positive, neutral and negative emotional load respectively $\mathrm{F}(2$, $56)=16.987, p<0.001]$ but no significant effect of mood $[522,512,514 \mathrm{~ms}$ for positive, neutral and negative emotional load respectively; $\mathrm{F}(2,56)=0.237, p=0.790]$ and interaction of mood and emotional load $(\mathrm{F}(4,112)=0.357, p=0.839$, Fig. 2a). Post hoc tests using the Bonferroni correction revealed that participants reacted significantly quicker to the words with negative emotional content than to positive $(p<0.001)$ and neutral ones $(p=0.001)$.

We then followed with a two-way repeated measures ANOVA for the accuracy of the performance in the 2-back task for emotional load conditions (positive, neutral, negative) as within-subjects factor and mood conditions (positive, neutral, negative) as between-subjects factor. This ANOVA revealed a significant effect of $\operatorname{mood}[77 \%, 68 \%, 69 \%$ for positive, neutral and negative mood respectively; $\mathrm{F}(2,56)=$ $6.202, p=0.004$ ] and a significant effect of emotional load $[65 \%, 75 \%, 74 \%$ for positive, neutral and negative emotional

a

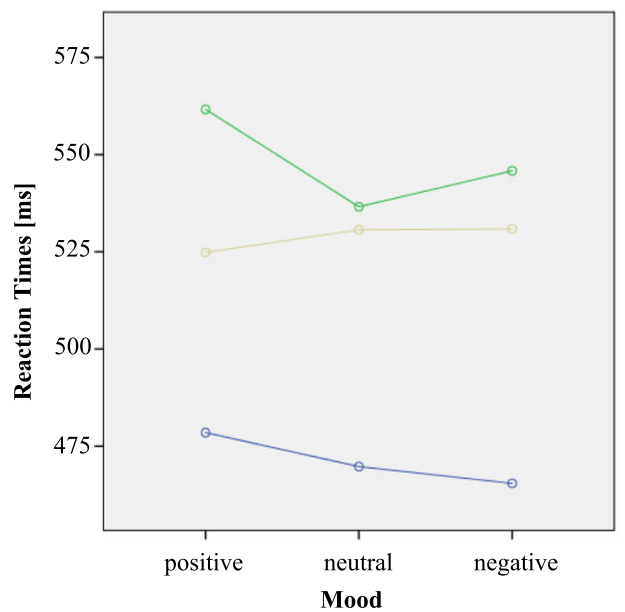

Fig. 2 a Reaction Times in the 2-back task for all mood conditions (positive, negative, neutral) and emotional load (positive, negative, neutral). The repeated measures ANOVA revealed a significant effect of the emotional load $(p<0.001)$ but no significant effect of mood or interaction of mood and emotional load ( $\mathrm{ps}>0.3$ ). b Accuracy of the performance in load respectively; $\mathrm{F}(2,56)=11.157, p<0.001]$ but we failed to obtain an interaction of mood and emotional load ( $\mathrm{F}(4$, $112)=1.740, p=0.146$; Fig. 2b). Post hoc tests using the Bonferroni correction revealed that participants reacted more accurately when being in a positive, compared to a negative $(p=0.036)$ and to neutral $(p=0.009)$ mood, and the least accurate when they processed words with positive emotional content compared to negative $(p=0.002)$ and to neutral ones $(p=0.001)$.

Distraction from the Mood We ran paired t-tests to measure the difference in the mood before and after performing the $n$ back task. As expected, a significant difference was found in the negative and positive conditions $(\mathrm{t}(29)=$ $-5.308, p<0.001, \mathrm{t}(29)=3.942, p<0.001)$, but not in the neutral one $(\mathrm{t}(29)=-0.797, p=0.432$; see Table 3$)$.

To further investigate whether the significant effect of the mood distraction after playing n-back was not caused by the direct impact of the emotional words that may have influenced mood (rather than the working memory load itself), we performed a two-way repeated measures ANOVA with mood condition (positive, neutral, negative) as between-subjects factor,

b

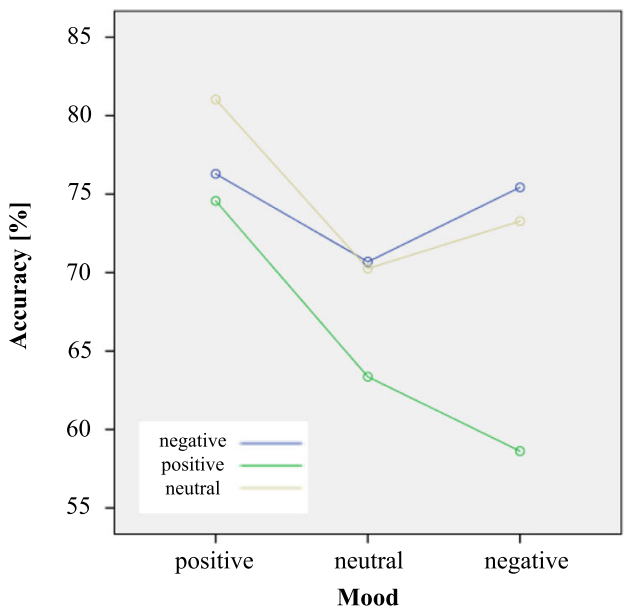

the 2-back task for all mood conditions (positive, negative, neutral) and emotional load (positive, negative, neutral). The repeated measures ANOVA revealed a significant effect of the emotional load $(p=0.004)$ and significant effect of mood $(p=0.002)$ but no significant interaction of mood and emotional load $(p>0.1)$ 
Table 3 Changes in the mood before and after performing $n$-back task all conditions. Participants answered the question: "Which mood state are you in now?" on a 7-point scale ( 0 - very negative to 6 - very positive). Significance levels:* $p<0.05, * * p<0.005, * * * p<0.001$

\begin{tabular}{|c|c|c|c|c|c|c|}
\hline \multirow[t]{2}{*}{ Mood State } & \multirow[t]{2}{*}{$\mathrm{N}$} & \multicolumn{2}{|l|}{$\mathrm{M}$} & \multicolumn{2}{|l|}{$\mathrm{SD}$} & \multirow[t]{2}{*}{$\mathrm{t}$} \\
\hline & & before & after & before & after & \\
\hline Negative mood before vs. after n-back & 30 & 2.77 & 3.80 & 0.63 & 0.92 & $-5.308 * * *$ \\
\hline Positive mood before vs. after n-back & 30 & 4.77 & 3.77 & 1.00 & 1.00 & $3.942 * * *$ \\
\hline Neutral mood before vs. after n-back & 30 & 3.57 & 3.77 & 0.68 & 1.04 & -0.797 \\
\hline
\end{tabular}

emotional load of the words (positive, neutral, negative) and task half (first, second) for the performance accuracy as within-subjects factors. This ANOVA revealed a significant effect of mood $[76 \%, 67 \%, 67 \%$ for positive, neutral and negative mood respectively $\mathrm{F}(2,58)=3.924, p=0.025$ ], a significant effect of emotional load [64\%, 73\%, 73\% for positive, neutral and negative emotional load respectively $\mathrm{F}(2,58)=12.652$, $p<0.001]$, and a significant effect of the task half [66\%, 74\% for first and second half, respectively $F(1,29)=16.338$, $p<0.001]$ but we failed to obtain any significant interactions. Post hoc tests using the Bonferroni correction revealed that participants reacted significantly better in the second half of the $n$-back task in comparison to the first $(p<0.001)$.

We followed with a two-way repeated measures ANOVA with mood condition (positive, neutral, negative) as betweensubjects factor, emotional load of the words (positive, neutral, negative) and task half (first, second) for reaction times (RTs) as within-subjects factors. This ANOVA revealed a significant effect of mood [398, 342, $344 \mathrm{~ms}$ for positive, neutral and negative mood respectively $\mathrm{F}(2,58)=4.322, p=0.018$ ], a significant effect of emotional load [353, 385, $346 \mathrm{~ms}$ for positive, neutral and negative emotional load respectively $\mathrm{F}(2,58)=$ $3.880, p=0.026]$, and a significant effect of the task half $(345,378 \mathrm{~ms}$ for first and second half respectively $\mathrm{F}(1,29)=$ 9.252, $p=0.005$ ) but we failed to obtain any significant interactions. Post hoc tests using the Bonferroni correction revealed that participants reacted significantly slower in the second half of the n-back task in comparison to the first $(p=0.005)$.

\section{Discussion}

In the present study, we argued that combining mood state with emotional words would have a significant, combined impact on the performance of the working memory. Our results show that, indeed, mood and emotional load have significant effect on the working memory performance, i.e. on the accuracy with which we react to emotional stimuli being in the certain mood and that working memory system would react with different speed dependently of the emotional content of words. However, our results speak in favour of the importance of the mood and emotions in processing daily events, they do not show a combined effect of mood and emotional content of the stimuli.
In our study participants were significantly worse in the 2back task when processing positive emotional words in contrary to neutral and negative ones, and significantly better when they were in the positive mood contrary to neutral and negative one. Moreover, participants responded much faster to negative emotional words than to other word-categories (positive, neutral), independent of their mood state.

Our results are in line with previous research. Internal affective cues, such as for example mood, direct the attention: positive mood focusing on the whole picture while negative mood - to the details (e.g., Easterbrook, 1959; Batson et al. 1992, Oaksford et al. 1996; Seibert and Ellis 1991; Spies et al. 1996; Gasper and Clore, 2002; Fredrickson and Branigan, 2005; Huntsinger, 2013; Sereno et al. 2015). These two trigger holistic and analytical thinking, respectively. In our study we show that words in the positive mood of the participants were processed significantly more accurately compared to other mood states. It might have been because positive mood triggered heuristic thinking, and less stressful environment for the participants, and in this case it facilitated the performance. However, participants processed the words with positive emotional content less accurately.

This result speaks in favour of the abovementioned hypotheses, that the positive mood facilitates working memory performance (Isen et al. 1987; Ashby et al. 1999). In this research paper, however, we also argued that, combination of emotional stimuli and mood would have a significant combined impact on the working memory system. Our finding may indicate that when the working memory system receives either ambiguous information (positive mood combined with negative stimuli), or simply receives too much simultaneous information (such as when the negative mood is combined with negative stimuli), it becomes overloaded, resulting in poor performance on the primary task. Another explanation is that emotions and moods, often occurring together, differ significantly, and are thus processed separately by the cognitive system. Our result may be considered in line with the "multiple-mechanisms" view of emotional awareness, which indicates that when emotional stimuli in the visual modality are perceived, at least three cortical areas are at play: the visual cortex, the substructures involved in emotional processing, and those associated with attention (for review see: Mitchell and Greening 2012). According to this view emotional stimuli gain not only priority 
in processing but also in attention. Therefore, in our experiment combining visually presented emotional stimuli with the mood state of the subjects may have overloaded the working memory system. On the one hand focussing on the emotional stimuli, but on the other directing attention according to the current mood state (i.e. positive mood would broaden the attention while negative narrow it down). This is also consistent with research showing that working memory has limited capacity (Orzechowski et al. 2009).

Our results also show that the words with negative emotional content would be processed much faster that other words, independently of the mood of the participants. Several recent studies support the notion of the "negativity bias", i.e. negative stimuli that elicit more rapid and more prominent reaction than non-negative stimuli (e.g. Vaish et al. 2008; Carretié et al. 2001). Consequently, negative stimuli gain attention and prioritized processing. For example, subjects look longer at the photos that present a negative situation or a negative facial expression (e.g. Fiske 1980) and blink more when processing negative words compared to positive ones (e.g. Fogarty and Stern 1989; Ohira 1996; Ohira et al. 1998).

Secondly, here, we replicated findings that indicate that a negative mood can be successfully replaced from the working memory system after a cognitive task is performed. Also, we observed that a significantly lower (neutral) mood was experienced by participants after performing the n-back task in the negative condition. What is particularly striking is that we managed to show that positive mood might be displaced as well. We propose that, the working memory system treats mood as an incoming stimuli, and regardless of its type, treats it according to the same principles as other incoming stimuli, and thus can be replaced from the working memory system. This might further suggest that both positive and negative mood operate according to the same principles.

Our finding fits well with neuropsychological studies showing that the more we activate the higher cognitive mechanisms of attention and controlled processing, the less we involve the brain regions responsible for processing emotions (Cohen et al. 2001; Hariri et al. 2000; Northoff et al. 2004), and vice versa (Bishop et al. 2004). We also show that distraction from both negative and positive moods is possible by simply performing a working memory task. As expected, participants reported a neutral mood after performing the n-back task in both the negative and positive conditions. According to the previously mentioned research, working memory tasks may not only be a distraction from a negative mood (Van Dillen and Koole 2007), but people may also reduce anger as a result of performing working memory tasks which act as distractors (Gerin et al. 2006; Rusting and NolenHoeksema 1998). The new incoming stimuli, while completing a working memory task, have to compete for working memory resources and attention. When more capacity is needed to perform another action in the working memory system, there is automatically less space for processing mood. However, according to our results, the positive mood may also be reduced in order to gain more capacity for other tasks.

This result may, however, raise a few concerns. We report the significant decrease in the manipulated mood after performing the n-back task. While at the beginning of the $n$ back procedure subjects were in a particular mood, at the end they reported a neutral one. Therefore, in principle it would be possible that, regardless of their initial state, their mood changed back to neutral within the first few trials of the nback task. However, if it were the case, we would not have obtained the main effect of the mood and emotional load on the working memory performance. However, while we show that the effect of the mood and emotional load was present during the experiment, it decreased in the second half of the nback task. Indeed, participants responded significantly slower in the second half of the experiment. Therefore it constitutes a further evidence of replacing mood from the working memory system, causing less confusion and less overload.

To the best of our knowledge, our study is the first of its kind to look at a range of emotional social situations (positive, negative, neutral) and working memory capacities while employing a highly controlled experimental manipulation of mood, with only one parameter that changed between the conditions (i.e. the number of balls being tossed in Cyberball). After playing in the ostracism condition (in our study referred to as negative mood condition), students reported significantly lower mood state - i.e. a negative one. Compared to the nonostracized students (in case of our study - those being in the positive and neutral mood condition), young adults being ostracized reported a significant decrease in their mood state after playing the game, replicating previous findings (Ruggieri et al. 2013). Our results correspond well to the previous research indicating that even short periods of being socially excluded by non-significant others may lead to a significant decrease in the mood state. Additionally, they show significant increase in the mood state when participants are socially included.

Although we have replicated some of the previous findings, our results did not clearly show what effect the combination of emotional words and moods has on the working memory. This discrepancy could stem from a number of different issues, including the application of the unique experimental paradigm we created. Although we have not entirely excluded the possibility that the mood itself decreased with time during performing the n-back task, the task itself lasted no longer than $15 \mathrm{~min}$. Since there is no intentional object that causes the mood state, the mood can last much longer than minutes. Thus under assumption of limited working memory capacity, we argue that mood, seen as an incoming information, was replaced from the system, by performing an n-back task. Nevertheless, further studies should be conducted to allow for a fuller understanding of the subject. 
The current results, however, support the view that mood and emotional content of words play a key role in processing incoming information, even though they do not interact with each other. Although emotions and mood often co-occur, they are usually mistaken for one another and have a significant impact on the working memory, but not a combined one. Small everyday events that influence mood may substantially impair or enhance the performance of working memory. Here, we have shown that not only we can manipulate mood without major difficulties (by simply doing a math task), but also that, at least partially, the nature of both positive and negative mood overlaps, despite the different neuronal networks at play. We also shed additional light on the working memory system, which seems to perceive both positive and negative moods in a similar way.

\section{Compliance with Ethical Standards}

Competing Interests On behalf of all authors, the corresponding author states that there is no conflict of interest.

Open Access This article is distributed under the terms of the Creative Commons Attribution 4.0 International License (http:// creativecommons.org/licenses/by/4.0/), which permits unrestricted use, distribution, and reproduction in any medium, provided you give appropriate credit to the original author(s) and the source, provide a link to the Creative Commons license, and indicate if changes were made.

\section{References}

Anderson, A. K., \& Phelps, E. A. (2001). Lesions of the human amygdala impair enhanced perception of emotionally salient events. Nature, 411, 305-309.

Ashby, F. G., Isen, A. M., \& Turken, A. U. (1999). A neuropsychological theory of positive affect and its influence on cognition. Psychological Review, 106, 529-550.

Batson, C. D., Shaw, L. L., \& Oleson, K. C. (1992). Differentiating affect, mood and emotion. In M. S. Clark (Ed.), Emotion (pp. 294-326). Newbury Park: Sage.

Bishop, S., Duncan, J., \& Lawrence, A. D. (2004). Prefrontal cortical function and anxiety: Controlling attention to threat-related stimuli. Nature Neuroscience, 7, 184-188.

Bower, G. H. (1981). Mood and memory. American Psychologist, 36, 129-148.

Carpenter, S. M., Peters, E., Vastfjall, D., \& Isen, A. M. (2007). The influence of mood on decision making in the aging adult. Presented at the SJDM.

Carretié, L., Mercado, F., Tapia, M., \& Hinojosa, J. A. (2001). Emotion, attention, and the 'negativity bias', studied through event-related potentials. International Journal of Psychophysiology, 41(1), 7585. https://doi.org/10.1016/S0167-8760(00)00195-1.

Cheng, P. W., \& Holyoak, K. J. (1985). Pragmatic versus syntactic approaches to training deductive reasoning. Cognitive Psychology, 17, 391-416.

Cohen, R., Lohr, I., Paul, R., \& Boland, R. (2001). Impairments of attention and effort among patients with major affective disorders. Journal of Neuropsychiatry and Clinical Neurosciences, 13, 385-395.

Conway, A. R., Jarrold, C., Kane, M. J., Akira, M., \& Towse, J. N. (2007). In A. R. Conway, C. Jarrold, M. J. Kane, M. Akira, \& J. N. Towse (Eds.), Ch. 1 Variation in Working Memory: An
Introduction in Variation in Working Memory (pp. 3-15). Oxford University Press.

Cowan, N. (2005). Working memory capacity. Hove. East Sussex: Psychology Press.

Dolan, R. J. (2000). Emotional processing in the human brain revealed through functional neuroimaging. In M. S. E. Gazzaniga (Ed.), The new cognitive neurosciences (pp. 1115-1132). Cambridge: MIT Press.

Donovan, J. K., \& Kleinknecht, P. D. (2007). Mood congruency as a function of emotion and priming. Western Psychological Association.

Easterbrook, J. A. (1959). The effect of emotion on cue utilization and the organization of behavior. Psychological review, 66(3), 183.

Eisenberger, N. I., Lieberman, M. D., \& Williams, K. D. (2003). Does rejection hurt? An fMRI study of social exclusion. Science, 302(5643), 290-292.

Eysenck, M. W., \& Calvo, M. (1992). Anxiety and performance: The processing efficiency theory. Cognition and Emotion, 6, 409-434.

Ferraro, F. R., King, B., Ronning, B., Pekarski, K., \& Risan, J. (2003). Effects of induced emotional state on lexical processing in younger and older adults. The Journal of Psychology, 137(3), 262-272.

Fiske, S. T. (1980). Attention and weight in person perception: The impact of negative and extreme behavior. Journal of Personality and Social Psychology, 38(6), 889-906.

Fogarty, C., \& Stern, J. A. (1989). Eye movements and blinks: Their relationship to higher cognitive processes. International Journal of Psychophysiology, 8(1), 35-42.

Forgas, J. P. (2000). Introduction: The role of affect in social cognition. In J. P. Forgas (Ed.), Feeling and thinking: The role of affect in social cognition (pp. 1-28). New York: Cambridge University Press.

Fredrickson, B. L., \& Branigan, C. (2005). Positive emotions broaden the scope of attention and thought-action repertoires. Cognition \& emotion, 19(3), 313-332.

Gasper, K., \& Clore, G. L. (2002). Attending to the big picture: Mood and global versus local processing of visual information. Psychological science, 13(1), 34-40.

Gerin, W., Davidson, K. W., Christenfeld, N. J. S., Goyal, T., \& Schwartz, J. E. (2006). The role of angry rumination and distraction in blood pressure recovery from emotional arousal. Psychosomatic Medicine, $68,64-72$.

Halberstadt, J. B., Niedenthal, P. M., \& Kushner, J. (1995). Resolution of lexical ambiguity by emotional state. Psychological Science, 6(5), 278-282.

Hariri, A. R., Bookheimer, S. Y., \& Mazziotta, J. C. (2000). Modulating emotional responses: Effects of a neocortical network on the limbic system. Neuroreport, 11, 43-48.

Huntsinger, J. R. (2013). Does emotion directly tune the scope of attention?. Current Directions in Psychological Science, 22(4), 265-270.

Isen, A. M. (1999). Positive affect. In T. Dagleish \& M. Powers (Eds.), The handbook of cognition and emotion (75-94). Hillsdale, NJ: Erlbaum.

Isen, A. M., Daubman, K. A., \& Nowicki, G. P. (1987). Positive affect facilities creative problem solving. Journal of Personality and Social Psychology, 52, 1122-1131.

Joormann, J., \& Siemer, M. (2004). Memory accessibility, mood regulation, and dysphoria: Difficulties in repairing sad mood with happy memories? Journal of Abnormal Psychology, 113, 179-188.

Mitchell, D. G., \& Greening, S. G. (2012). Conscious perception of emotional stimuli: Brain mechanisms. The Neuroscientist, 18(4), 386-398.

Morrow, J., \& Nolen-Hoeksema, S. (1990). Effects of responses to depression on the remediation of depressive affect. Journal of Personality and Social Psychology, 58, 519-527.

Niedenthal, P. M., \& Setterlund, J. B. H. M. B. (1997). Being happy and seeing "happy": Emotional state mediates visual word recognition. Cognition \& Emotion, 11(4), 403-432. 
Northoff, G., Heinzel, A., Bermpohl, F., Niese, R., Pfennig, A., PascualLeone, A., \& Schlaug, G. (2004). Reciprocal modulation and attenuation in the prefrontal cortex: An fMRI study on emotionalcognitive interaction. Human Brain Mapping, 21, 202-212.

Oaksford, M., Morris, F., Grainger, B., \& Williams, J. M. G. (1996). Mood, reasoning, and central executive processes. Journal of Experimental Psychology: Learning, Memory, and Cognition, 22, 477-493.

Ohira, H. (1996). Eyeblink activity in a word-naming task as a function of semantic priming and cognitive load. Perceptual and Motor Skills, 82(3), 835-842.

Ohira, H., Winton, W. M., \& Oyama, M. (1998). Effects of stimulus valence on recognition memory and endogenous eyeblinks: Further evidence for positive-negative asymmetry. Personality and Social Psychology Bulletin, 24(9), 986-993.

Olafson, K. M., \& Ferraro, F. R. (2001). Effects of emotional state on lexical decision performance. Brain and Cognition, 45(1), 15-20.

Orzechowski, J., Piotrowski, K. T., Balas, R. i., \& Stettner, Z. (2009). Pamięć Robocza. Warszawa: Wydawnictwo SWPS Academica.

Peirce, J. W. (2009). Generating stimuli for neuroscience using PsychoPy. Frontiers in Neuroinformatics, 2(10), 1-8. https://doi.org/10.3389/ neuro.11.010.2008

Phillips, L. H., Bull, R., Adams, E., \& Fraser, L. (2002). Positive mood and executive function: Evidence from Stroop and fluency tasks. Emotion, 2, 21-22.

Pratto, F., \& John, O. P. (1991). Automatic vigilance: The attentiongrabbing power of negative social information. Journal of Personality and Social Psychology, 61, 380-391.

Reimann, B., \& McNally, R. (1995). Cognitive processing of personally relevant information. Cognition and Emotion, 9, 324-340.

Ruggieri, S., Bendixen, M., Gabriel, U., \& Alsaker, F. (2013). Cyberball. Swiss Journal of Psychology, 72, 103-109.

Rusting, C. L., \& Nolen-Hoeksema, S. (1998). Regulating responses to anger: Effects of rumination and distraction on angry mood. Journal of Personality and Social Psychology, 74, 790-803.

Seibert, P. S., \& Ellis, H. C. (1991). Irrelevant thoughts, emotional mood states, and cognitive task performance. Memory \& Cognition, 19, 507-513.
Sereno, S. C., Scott, G. G., Yao, B., Thaden, E. J., \& O'Donnell, P. J. (2015). Emotion word processing: Does mood make a difference? Frontiers in Psychology, 6, 1191.

Small, S. A. (1985). The effect of mood on word recognition. Bulletin of the Psychonomic Society, 23(6), 453-455.

Spies, K., Hesse, F. W., \& Hummitzsch, C. (1996). Mood and capacity in Baddeley's model of human memory. Zeitschrift fur Psychologie, 204, 367-381.

Tabert, M. H., Borod, J. C., Tang, C. Y., Lange, G., Wei, T. C., Johnson, R., Nusbaum, A. O., \& Buchsbaum, M. S. (2001). Differential amygdale activation during emotional decision and recognition memory tasks using unpleasant words: An fMRI study. Neuropsychologia, 39, 556-573.

Teasdale, J. D. (1983). Negative thinking in depression: Cause, effect, or reciprocal relationship? Advances in Behaviour Research and Therapy, 5, 3-25.

Teasdale, J. D., \& Fogarty, S. J. (1979). Differential effects of induced mood on retrieval of pleasant and unpleasant events from episodic memory. Journal of Abnormal Psychology, 88, 248-257.

Vaish, A., Grossmann, T., \& Woodward, A. (2008). Not all emotions are created equal: The negativity bias in social-emotional development. Psychological Bulletin, 134(3), 383-403.

Van Dillen, L. F., \& Koole, S. L. (2007). Clearing the mind: A working memory model of distraction from negative mood. Emotion, 7(4), 715-723.

Williams, K. D. (2007). Ostracism: The kiss of social death. Social and Personality Psychology Compass, 1(1), 236-247.

Williams, K. D., \& Jarvis, B. (2006). Cyberball: A program for use in research on interpersonal ostracism and acceptance. Behavior Research Methods, 38(1), 174-180.

Williams, K. D., Cheung, C. K. T., \& Choi, W. (2000). Cyberostracism: Effects of being ignored over the internet. Journal of Personality and Social Psychology, 79(5), 748-762.

Publisher's note Springer Nature remains neutral with regard to jurisdictional claims in published maps and institutional affiliations. 